\title{
The initial phase of chromosome condensation requires Cdk1-mediated phosphorylation of the CAP-D3 subunit of condensin II
}

\author{
Satoshi Abe, ${ }^{1,2,6}$ Kota Nagasaka, ${ }^{1,3,6}$ Youko Hirayama, ${ }^{1}$ Hiroko Kozuka-Hata, ${ }^{4}$ Masaaki Oyama, ${ }^{4}$ \\ Yutaka Aoyagi, ${ }^{2}$ Chikashi Obuse, ${ }^{5}$ and Toru Hirota ${ }^{1,3,7}$ \\ ${ }^{1}$ Cancer Institute, Japanese Foundation for Cancer Research (JFCR), Tokyo 135-8550, Japan; ${ }^{2}$ Niigata University Graduate School \\ of Medical and Dental Sciences, Niigata 951-8122, Japan; ${ }^{3}$ Graduate School of Bioscience and Biotechnology, Tokyo Institute of \\ Technology, Yokohama 226-8501, Japan; ${ }^{4}$ Institute of Medical Science, University of Tokyo, Tokyo 108-8639, Japan; ${ }^{5}$ Graduate \\ School of Life Science, Hokkaido University, Sapporo 001-0021, Japan
}

The cell cycle transition from interphase into mitosis is best characterized by the appearance of condensed chromosomes that become microscopically visible as thread-like structures in nuclei. Biochemically, launching the mitotic program requires the activation of the mitotic cyclin-dependent kinase Cdk1 (cyclin-dependent kinase 1), but whether and how Cdk1 triggers chromosome assembly at mitotic entry are not well understood. Here we report that mitotic chromosome assembly in prophase depends on Cdk1-mediated phosphorylation of the condensin II complex. We identified Thr 1415 of the CAP-D3 subunit as a Cdk1 phosphorylation site, which proved crucial as it was required for the Polo kinase Plk1 (Polo-like kinase 1) to localize to chromosome axes through binding to CAP-D3 and thereby hyperphosphorylate the condensin II complex. Live-cell imaging analysis of cells carrying nonphosphorylatable CAP-D3 mutants in place of endogenous protein suggested that phosphorylation of Thr 1415 is required for timely chromosome condensation during prophase, and that the Plk1mediated phosphorylation of condensin II facilitates its ability to assemble chromosomes properly. These observations provide an explanation for how Cdk1 induces chromosome assembly in cells entering mitosis, and underscore the significance of the cooperative action of Plk1 with Cdk1.

[Keywords: cyclin-dependent kinase 1; condensin; mitosis; Polo-like kinase 1; prophase]

Supplemental material is available for this article.

Received November 26, 2010; revised version accepted March 2, 2011.

Cells pass their genome from one to the next by forming mitotic chromosomes that become discernible as cells divide. Such large-scale reorganization of chromatin fibers into mitotic chromosomes depends on chromosome condensation and dissociation of sister chromatid cohesion. These two processes, together constituting mitotic chromosome assembly, initiate in prophase and are thought to ensure the correct orientation of chromosomes on the mitotic spindle and the faithful separation of sister chromatids in anaphase. The mitotic cyclindependent kinase 1 (Cdk1) has a fundamental function to drive cells into mitosis, as cells, in principle, do not initiate the mitotic entry program in the absence of Cdk1 activity (Nurse 1990; Nigg 1995). Despite its undisputed role in inducing the mitotic program, surprisingly few

\footnotetext{
${ }^{6}$ These authors contributed equally to this work.

${ }^{7}$ Corresponding author.

E-MAIL thirota@jfcr.or.jp; FAX 81-3-3570-0354.

Article is online at http://www.genesdev.org/cgi/doi/10.1101/gad.2016411.
}

substrates for Cdk1 have been identified to date. Thus, finding direct substrates for Cdk1 in this context continues to be a major challenge in order to elucidate the molecular mechanisms underlying morphological changes as cells enter mitosis, including chromosome assembly.

Mitotic chromosome assembly is mediated primarily through the activity of Smc (structural maintenance of chromosomes) protein complexes called condensins (for review, see Hirano 2005; Nasmyth and Haering 2005). Two classes of condensin complex, termed condensin I and II, have been identified in metazoans that share the core two ATPases-Smc2 and Smc 4 (also known as CAP-E and CAP-C, respectively) - and contain different, yet related, sets of non-Smc subunits. In human cells, nonSmc subunits of condensin I include CAP-D2, CAP-G, and CAP-H (also known as Kleisin- $\gamma$ ), and the respective counterparts in the condensin II complex are called CAPD3, CAP-G2, and CAP-H2 (also known as Kleisin- $\beta$ ) (Ono et al. 2003; Yeong et al. 2003). Despite these structural similarities, condensin I and condensin II behave differently 
from each other and have distinct functions during mitotic chromosome assembly (Hirota et al. 2004; Ono et al. 2004). Condensin I localizes in the cytoplasm until the nuclear envelope breakdown (NEBD), and gains access to chromosomes thereafter to confer physical properties to chromosomes required to withstand spindle-pulling forces (Gerlich et al. 2006). Condensin II, in contrast, localizes in the nucleus and thus can accumulate at chromosome axes from early stages of prophase. Consistent with this observation, the activity of condensin II is indispensable to initiate chromosome condensation in prophase (Hirota et al. 2004; Ono et al. 2004).

The activity of condensin is stimulated during mitosis when condensin subunits are phosphorylated (for review, see Hirano 2009|. Seminal experiments using frog egg extracts showed that the activity of condensin, quantified by measuring its ability to induce supercoils into DNA substrates, is stimulated during mitosis when condensin I subunits are phosphorylated by Cdk1 (Kimura et al. 1998). The requirement of another major mitotic kinase, Aurora B, for the association of condensin I to chromosomes is widespread in metazoans (Giet and Glover 2001; Hagstrom et al. 2002; Kaitna et al. 2002; Petersen and Hagan 2003; Lipp et al. 2007; Takemoto et al. 2007). Similarly, in budding yeast, whose single class of condensin is structurally related to vertebrate condensin I, condensin is phosphorylated by the Aurora kinase Ipl1 (Lavoie et al. 2004). Moreover, in this organism, the Polo kinase Cdc5 has been found recently to phosphorylate condensin and promote supercoiling activity and chromosome condensation in anaphase (St-Pierre et al. 2009). In contrast to what had been unraveled for condensin I regulation, it is largely unknown how condensin II is controlled, however.

What are the mechanisms that drive condensation of the duplicated chromosomes as the cell enters mitosis? Because condensin II is the only chromosomal component that is known to be essential for timely chromosome condensation in prophase (Hirota et al. 2004; Ono et al. 2004), elucidating the regulation of condensin II would provide a clue to this long-standing question. Here we described that chromosome condensation in prophase depends on Cdk1-mediated phosphorylation of the CAP-D3 subunit of condensin II. We found that Cdk1 phosphorylation of the Thr 1415 residue allows Plk1 (Polo-like kinase 1) recruitment to CAP-D3 and the chromosomal axis, which then propagates phosphorylation of the whole condensin II complex. Replacement of endogenous CAPD3 by nonphosphorylatable mutants indicated that the phosphorylation of Thr 1415 triggers chromosome condensation in prophase and stimulates condensin II-mediated assembly of chromosomes. Defining CAP-D3 as a physiological substrate of Cdk1 provides a molecular explanation for how Cdk1 triggers mitotic chromosome assembly.

\section{Results}

Plk1 binds to the condensin II complex

To address how condensin II is regulated in mitosis, we searched for proteins that bind to condensin II in mitotic cell extracts. Mass spectrometric analysis of immunoprecipitates using antibodies to the condensin II subunit CAP-D3 identified the Polo kinase Plk1 as a candidate binding protein of condensin II. To validate this interaction, we performed an immunoprecipitation assay using different polyclonal antibodies to condensin II subunits. All of the antibodies used immunoprecipitated Plk1 as well as condensin II components from mitotic cell extracts (Supplemental Fig. 1A). Coimmunoprecipitation of Plk1 was seen more clearly when condensin II complexes were purified from a mitotic chromosomeenriched fraction, implying that the interaction occurs predominantly on chromosomes (Fig. 1A).

One mode of interaction of Plk1 with its binding proteins is via a phospho-Ser/Thr-binding motif called the polo-box domain (PBD) (Elia et al. 2003). To address whether Plk1 binds directly to condensin II-and, if so, which of the condensin subunits binds to Plk1-the condensin II complex purified from mitotic cell extracts was subjected to Far-Western analysis using recombinant PBD protein as a probe. We found a PBD signal on the CAP-D3 protein but not on the other subunits (Fig. 1B). The signal appeared to react specifically to a phosphorylated form of CAP-D3, which was revealed by retarded electrophoretic mobility in a polyacrylamide gel (Yeong et al. 2003; Lipp et al. 2007). Supporting this idea, this binding of PBD was diminished when isolated condensin II was pretreated with protein phosphatase (Fig. 1B). These data suggested that Plk1 binds directly to CAPD3 in a phosphorylation-dependent manner.

\section{Mitotic phosphorylation of condensin II depends on both Cdk1 and Plk1}

It has been shown that non-Smc subunits of condensin are phosphorylated in mitosis, some of which can be indicated by their electromobility shifts (Fig. 1C; Yeong et al. 2003). To be able to detect phosphorylated species more clearly, we used a Phos-tag acrylamide gel mixture for SDS-PAGE, which allowed us to differentiate the mobility shifts for all condensin non-Smc subunits (Fig. 1D). We then addressed which of the mitotic kinases are involved in the phosphorylation of condensin II. We found that a 20-min pretreatment of mitotic cells with a Cdk1 inhibitor abolished the mitotic mobility retardation of both condensin I and condensin II subunits. Plk1 inhibition largely reversed the mobility shifts for condensin II subunits. Among them, the mitotic retardation of CAP-D3 was markedly suppressed by Plk1 inhibition, implying that hyperphosphorylation of this protein depends on Plk1 activity, but some shift-up still remained, which appeared to depend on Cdk1 activity (Supplemental Fig. 1B). In contrast, Plk1 inhibition did not detectably affect the mitotic mobility retardation for condensin I subunits (Fig. 1D). These experiments suggested that mitotic phosphorylation of condensin II depends on both Cdk1 and Plk1. Consistent with this idea, a largescale proteomic analysis has recently found condensin II subunits as Plk1 substrates (Santamaria et al. 2011).

To investigate how these kinases are involved in regulating condensin II, we first generated cell lines that 
A
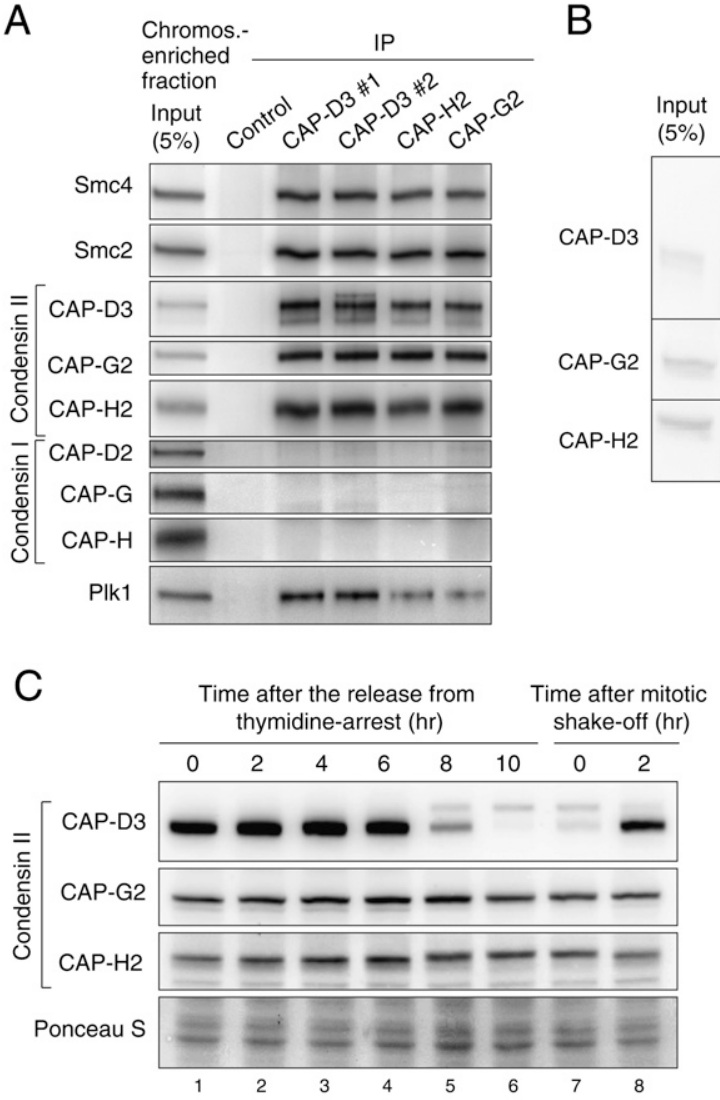

B
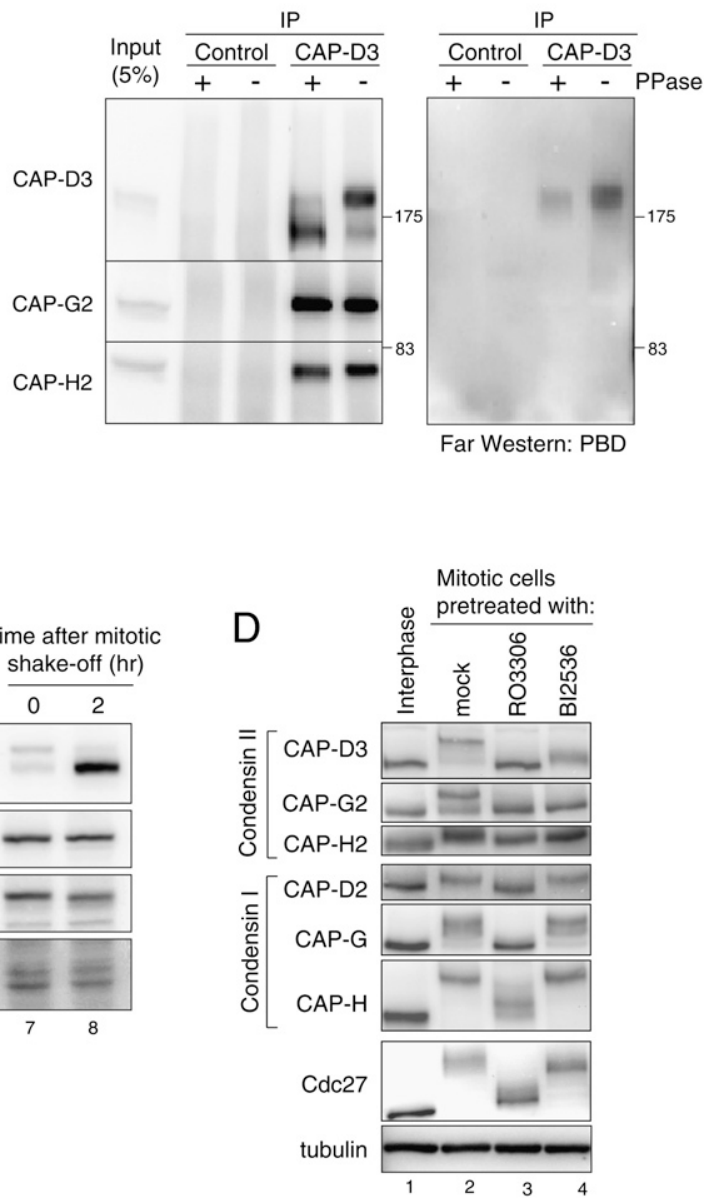

Figure 1. Regulation of condensin II in mitosis. (A) Immunoprecipitation of Plk1 with condensin II. Condensin II components were immunoprecipitated from a chromosome-enriched fraction prepared from mitotic cell extracts using either of two different antibodies to CAP-D3 (\#1 or \#2), CAP-G2, CAP-H2, or nonimmune IgG as a control, and resulting precipitates (IP) were analyzed by immunoblotting with the antibodies indicated. Note that none of the condensin I subunits copurified with the condensin II complex, discounting the possibility that the interaction between condensin II and Plk1 is mediated indirectly by DNA. $(B)$ Plk1 binds directly to CAP-D3 in a phosphorylation-dependent manner. Immunoprecipitates with either control or CAP-D3 antibodies from mitotic cell extracts were incubated with or without phosphatase (PPase), and were analyzed by immunoblotting with indicated antibodies (left panel) and Far-Western blotting with PBD (right panel). (C) Detection of CAP-D3 phosphorylation in mitotic cell extracts. Synchronized cell populations were analyzed by immunoblotting at the indicated times after the release from thymidine arrest (i.e., early $S$ phase) (lanes 1-6) or nocodazole arrest (i.e., mitosis) (lanes 7,8). As reported previously (Yeong et al. 2003; Lipp et al. 2007), slowly migrating bands of CAP-D3 were detected specifically during mitosis, but little retardation was detectable for CAP-G2 and CAP-H2. (D) Mitotic phosphorylation of condensin II depends on Cdk1 and Plk1. Thymidine-arrested interphase cells (lane 1), nocodazole-arrested mitotic cells (lane 2), a Cdk1 inhibitor RO3306-treated nocodazole-arrested mitotic cell (lane 3), or a Plk1 inhibitor BI2536-treated nocodazolearrested mitotic cell (lane 4) were analyzed by immunoblotting for condensin subunits. The mobility shifts for Cdc 27 verify that these pretreatments efficiently abolished the activities of the targeted kinases in mitotic cells (Kraft et al. 2003).

express GFP-tagged CAP-D3 at physiological levels, which appeared to possess tested properties of the endogenous protein (Supplemental Fig. 2A-C). We could also show that the GFP-tagged protein is functional, as the cell line expressing GFP-CAP-D3 showed a rescue of phenotypic defects observed after suppression of the endogenous gene by RNAi in analyses below. The finding that the association of Plk1 with CAP-D3 requires phosphorylation of CAP-D3 led us to predict that CAP-D3 contains the PBD-binding motif S-pS/pT-P (Elia et al. 2003). To obtain an objective picture of CAP-D3 phosphorylation, we performed mass spectrometric analysis of immunopurified CAP-D3 from mitotic cells (Supplemental Fig. 3). Among the phosphorylated residues suggested, Ser 1329 and Thr 1415 were found to meet the consensus of the PBD-binding motif.

In human CAP-D3, there are three sites in total that meet the PBD-binding motif, including the above two. To address whether any of these candidate sites are involved in the interaction with Plk1, we generated cell lines that express a series of GFP-tagged nonphosphorylatable mutants of full-length CAP-D3 by changing one of the 
candidate residues to alanine. By immunoprecipitation assay, we found that the binding of Plk1 was abolished when Thr 1415 is mutated (Fig. 2A). Consistent with this result, a Far-Western analysis revealed that the PBD protein did not bind to the Thr 1415 mutant when the others do (Fig. 2B). These results suggest that the binding of Plk1 to CAP-D3 depends on the phosphorylation of Thr 1415. Interestingly, the Thr 1415 residue appears to be conserved in higher eukaryotes, whereas the other two candidate sites are not (Fig. 2C).

Because phosphorylation of CAP-D3 depends on both Cdk1 and Plk1 (Fig. 1D), we thought one of these kinases might mediate CAP-D3 Thr 1415 phosphorylation, as these kinases are known to mediate phosphorylation of the PBD-binding motif (Elia et al. 2003; Petronczki et al. 2008; Archambault and Glover 2009). Moreover, we found that the amino acid sequence adjacent to Thr 1415 fits the consensus for Plk1, which led us to predict the Ser 1419 site as a target of this kinase (Fig. 2C). To address these possibilities, we generated recombinant polypeptides that encompass these prospective phospho-sites and used them as substrates for in vitro kinase reactions. The phosphorylation of the peptide was readily catalyzed by Cdk1/Cyclin B and Plk1. In these assays, phosphorylation by Cdk1/Cyclin B was significantly suppressed in a peptide bearing the T1415A substitution, and phosphorylation by Plk1 was suppressed in a S1419A peptide, suggesting that Cdk1 and Plk1 can mediate phosphorylation of CAP-D3 on the Thr 1415 and Ser 1419 residues, respectively (Fig. 2D). Notably, in addition to Thr 1415 , Ser 1419 or its equivalent site also seems to be conserved (Fig. 2C).

\section{Enrichment of Plk1 on chromosome axes}

The biochemical analyses suggest that Plk1 associates with condensin II by binding directly to CAP-D3. To address at which subcellular region this interaction might occur, we revisited the localization of Plk1 by immunofluoresence microscopy using monoclonal antibodies to Plk1, the specificity of which was verified by staining the Plk1-depleted cells with the same antibodies (Supplemental Fig. 4A-C). In addition to the characteristic localization of Plk1 at kinetochores and the central spindle, enrichment of Plk1 was evident on chromosomal axes during the early stages of mitosis (Fig. 3A), as suggested previously (Santamaria et al. 2007). This localization of Plk1 on chromosome arms accordingly colocalized with condensin II (Fig. 3C). Importantly, the localization of Plk1 on chromosomal axes was perturbed in the absence of CAP-D3, while its enrichment at kinetochores and the central spindle was not affected (Fig. 3B). With a closer inspection of chromosome spread preparations, the enrichment of Plk1 to chromosome axes was unambiguously
A

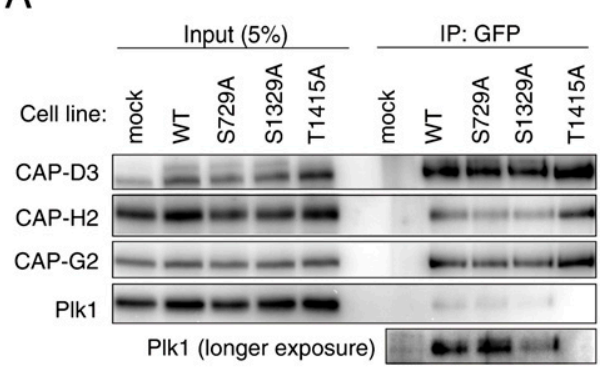

C
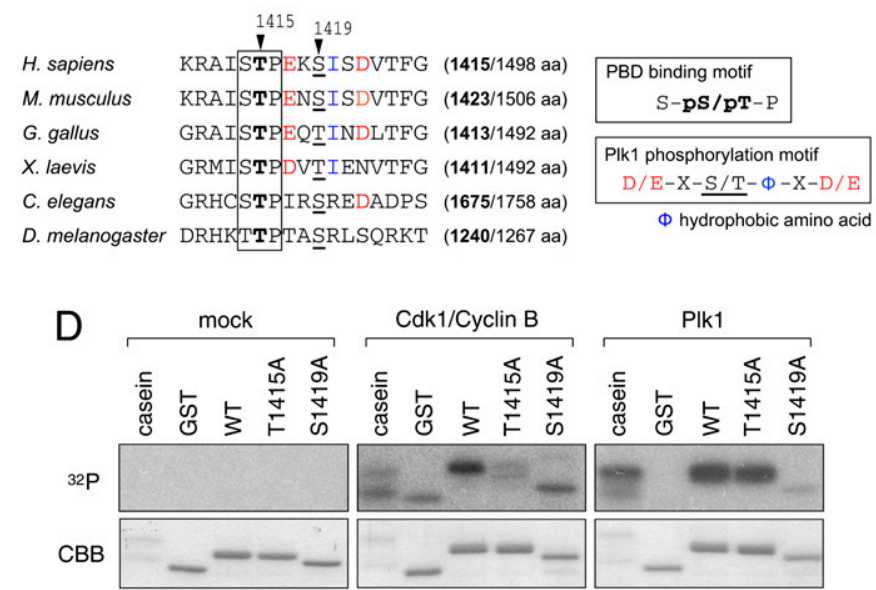

B

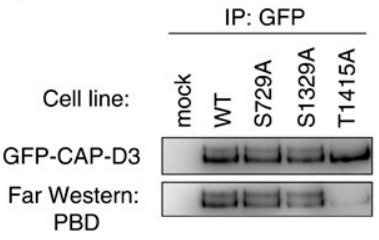

Figure 2. Identification of phosphorylation sites on CAP-D3. (A) Immunoprecipitation of Plk1 with CAP-D3 is abolished in a Thr 1415 nonphosphorylatable mutant. Mitotic cell extracts prepared from cells stably expressing the indicated version of GFPtagged CAP-D3 were subjected to immunoprecipitation analysis with antibodies to GFP and were immunoblotted with the antibodies indicated. $(B)$ The binding of PBD to CAP-D3 is lost in the T1415A mutant. The indicated series of GFP-tagged CAP-D3 proteins were immunoprecipitated (top panel) and analyzed by Far-Western analysis with PBD (bottom panel). Note that, like the endogenous protein, GFPCAP-D3 can be detected as two major bands in mitotic extracts from the wild-type and S729A and S1329A mutant cells, but not the T1415A cells. $(C)$ A PBD-binding motif in CAP-D3. Among three candidate sites, Thr 1415 and Ser 1419 fall within a PBD-binding motif (boxed) and the Plk1 consensus phosphorylation site (color-coded), respectively. Equivalent regions from orthologous proteins from different species are aligned, highlighting the evolutionary conservation of these motifs. The numbers in brackets indicate positions of the Thr 1415equivalent threonine (bold) in the full amino acid length of CAP-D3 protein. (D) Cdk1 and Plk1 mediate phosphorylation of Thr 1415 and Ser 1419, respectively, in vitro. A series of polypeptides corresponding to a partial fragment of CAP-D3 that encompasses the prospective phosphorylation sites were incubated with mock (control), Cdk1/Cyclin B,

or Plk1. (Top panels) Incorporation of ${ }^{32} \mathrm{P}$ was detected by autoradiography. (Bottom panels) Coomassie Brilliant Blue staining (CBB) verifies that equivalent amounts of substrate appear in each lane. 
A

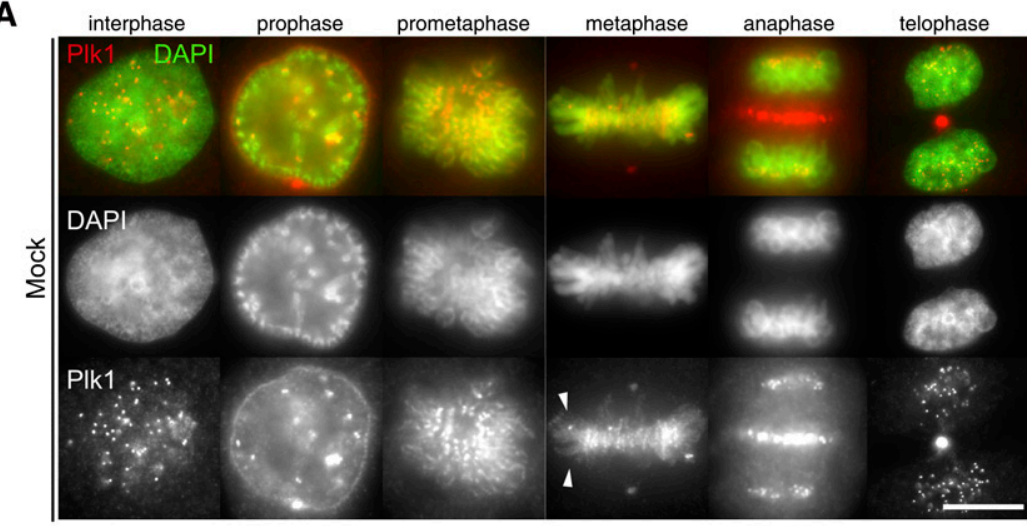

B

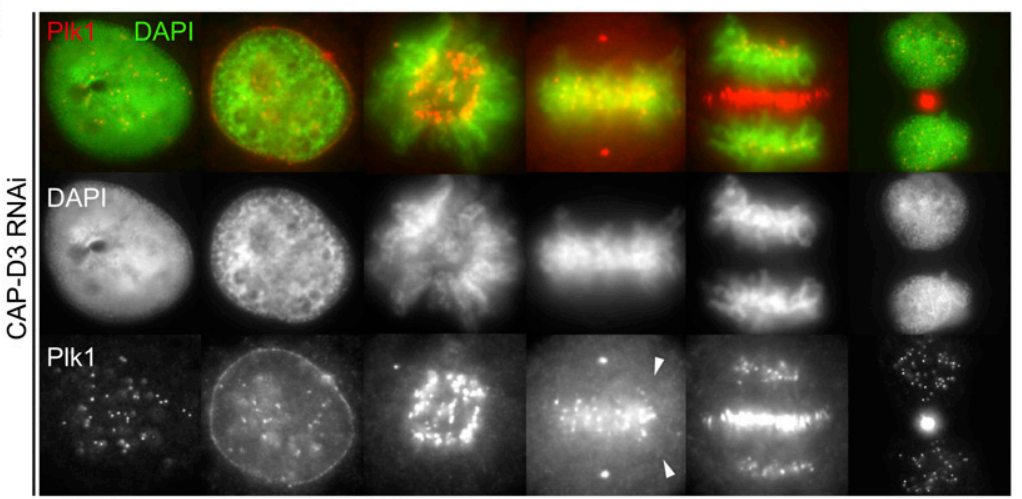

C

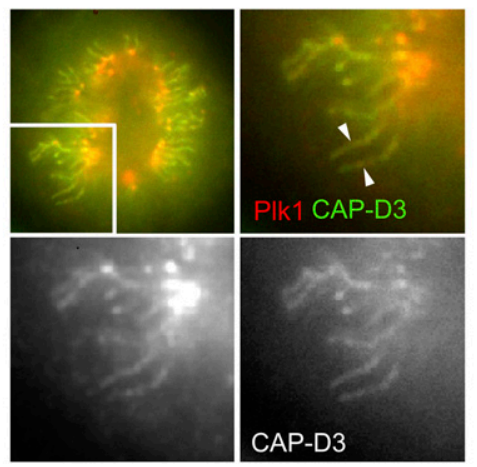

D

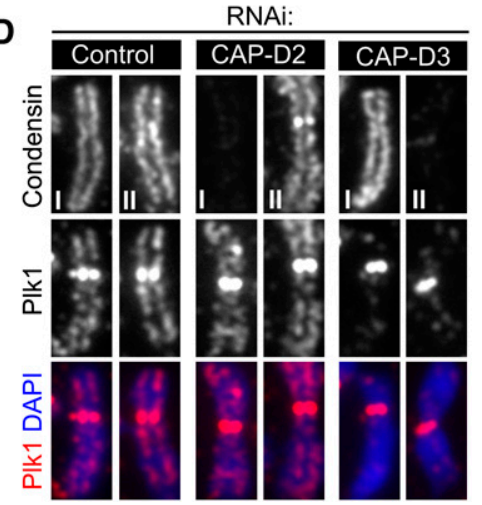

Figure 3. Enrichment of Plk1 on chromosomal axes. (A) Localization of Plk1 by immunofluorescence microscopy. Exponentially growing HeLa cells were fixed with methanol, incubated with antibodies to Plk1, and labeled with an Alexa fluorescent dye (red). DNA was stained with DAPI (green). Representative cells in interphase, prophase, prometaphase, metaphase, anaphase, and telophase are shown. Note the chromosome axial staining with Plk1 antibodies in prometaphase and metaphase (arrowheads). Bar, $10 \mu \mathrm{m}$. $(B)$ Enrichment of Plk1 on chromosome axes is lost in the absence of CAP-D3. Cells depleted of CAP-D3 were processed for immunofluorescence microscopy as in $A$. Note that Plk1 signals on chromosome axes are displaced (arrowheads), while the other localizations are preserved. $(C)$ Colocalization of Plk1 with CAP-D3. Wild-type GFP-CAP-D3-expressing cells were fixed and stained with Plk1 antibodies. Both CAP-D3 (right bottom panel; green in top merged panels) and Plk1 (left bottom panel; red in top merged panels) signals are visible on chromosome axes. (D) Enrichment of Plk1 at chromosome axes depends on condensin II but not condensin I. Chromosome spread samples prepared from mitotic cells that had been depleted of CAP-D2, CAP-D3, or mock (control) were fixed and stained for Plk1 with either condensin I (CAP-H) or condensin II (CAP-D3), as indicated. Quantification of fluorescence intensities for Plk1 signals is shown in Supplemental Figure 5A.

seen and abolished in CAP-D3-depleted cells but not in cells depleted of a condensin I subunit, CAP-D2 (Fig. 3D).

To address whether this CAP-D3-dependent chromosomal axis localization of Plk1 requires phosphorylation at Thr 1415, as the above biochemical assays suggested (Fig. 2 ), we generated a series of cell lines that stably express GFP-tagged nonphosphorylatable alanine mutants, and depleted endogenous CAP-D3 proteins from those cells. The immunoblot analysis of cell extracts showed that the endogenous protein was largely replaced with GFP-tagged CAP-D3 for each cell line (Fig. 4A). Under these conditions, we found that the chromosomal axis-enriched Plk1 became undetectable in T1415A-replaced cells, whereas wild-type-replaced or S1419A-replaced cells retained the axial Plk1 staining (Fig. 4B, arrowheads). These findings were further confirmed by immunofluoresence microscopy of spread chromosomes (Fig. 4C).

\section{Regulation of CAP-D3 phosphorylation in vivo}

Thus, the recruitment of Plk1 to chromosomal axes depends on the binding to CAP-D3, and crucial to this is the phosphorylation of this subunit on the Thr 1415 residue. To elucidate the regulation of CAP-D3 phosphorylation in vivo, we generated antibodies named pT1415 and pS1419, which can primarily recognize CAP-D3 when Thr 1415 or Ser 1419 residues are phosphorylated, respectively. Using these antibodies for immunoblot analyses, we found two phosphorylated species of CAP-D3 in mitotic cell extract; phosphorylation at Thr 1415 is seen in both bands, whereas phosphorylation at Ser 1419 contributes only in the upper band; i.e., the hyperphosphorylated form (Fig. 4D). As generation of the hyperphosphorylated form of CAP-D3 depends on the Plk1 activity (Fig. 1D), this finding implies that Ser 1419 is a phospho-site of Plk1. 
Abe et al.

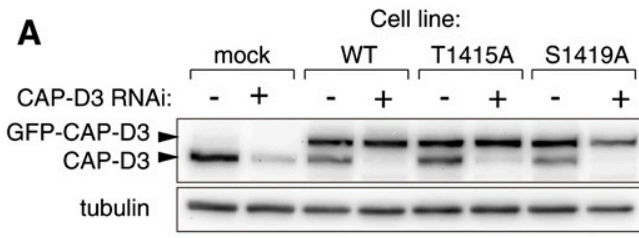

B

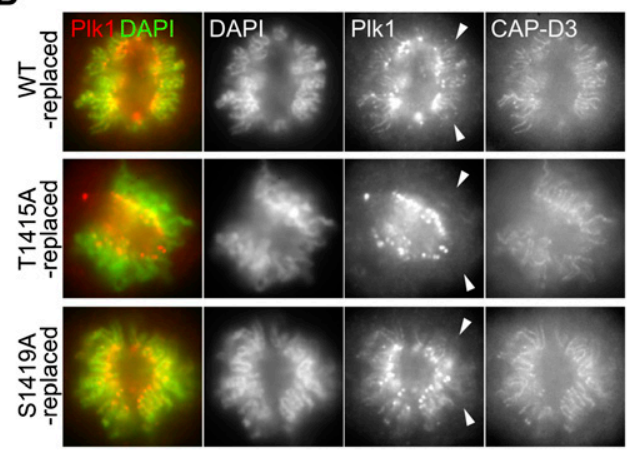

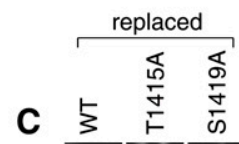

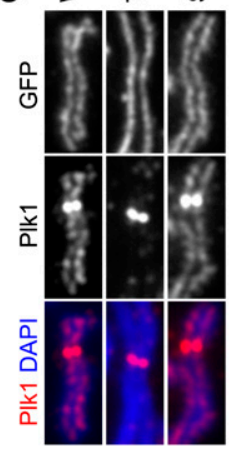

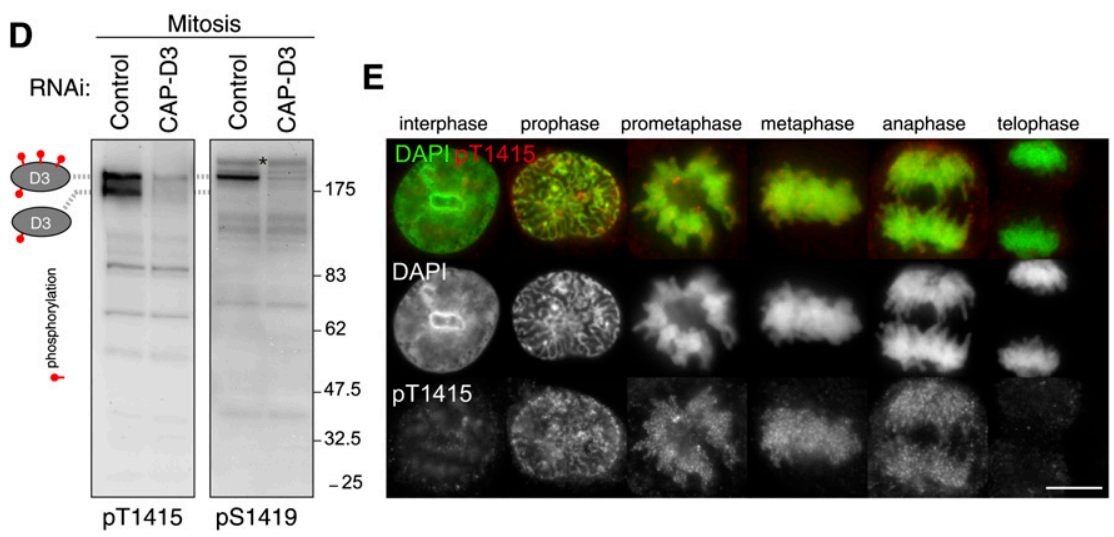

Figure 4. Mitotic phosphorylation of CAPD3 on Thr 1415 and Ser 1419. (A) Replacement of endogenous CAP-D3 protein with GFP-tagged CAP-D3 proteins. HeLa cells that stably express GFP-tagged GFP-D3, either wild-type (WT) or the nonphosphorylatable mutant for Thr 1415 (T1415A) or Ser 1419 (S1419A), were generated. Expression of endogenous CAP-D3 was suppressed by RNAi designed to target the untranslated region of the gene. Interphase cell extracts were analyzed by immunoblotting for the amount of endogenous CAP-D3 (bottom bands) and GFP-CAP-D3 (top bands). (B) Delocalization of Plk1 from chromosome axes in T1415A-replaced cells. Wild-typereplaced, T1415A-replaced, or S1419Areplaced cells were fixed and stained with Plk1 antibodies (red). DNA was stained with DAPI (green). Representative prometaphase cells are shown. Note that T1415A and S1419A were both found enriched at chromosomal axes at levels comparable with the wild-type version, which discounts the possibility that these phosphorylations have a major role in the chromosomal association of condensin II. (C) Enrichment of Plk1 at chromosome axes is perturbed in T1415Areplaced cells. Fixed chromosome spread samples prepared from mitotic cells that had been replaced with the indicated version of CAP-D3 were stained with Plk1 antibodies. Quantification of fluorescence intensities for Plk1 signals is shown in Supplemental Figure 5B. (D) Generation of phospho-specific CAP-D3 antibodies. Total extracts of nocodazole-arrested cells treated with either mock (control) or CAP-D3 RNAi

were subjected to immunoblotting by pT1415 or pS1419 antibodies. Note that two major pT1415 reactive bands and one major pS1419 reactive band (as denoted on the left side) are diminished in CAP-D3-depleted cells. Bands that do not disappear after CAP-D3 depletion are nonspecific reactive proteins (a band marked by an asterisk, for example). Specific reactivity to phospho-sites and mitotic forms are later shown in Supplemental Figure 6, A and B, respectively. (E) Timing of CAP-D3 Thr 1415 phosphorylation during mitotic progression. Fixed HeLa cells were stained with pT1415 antibodies and labeled with an Alexa dye (red). DNA was stained with DAPI (green). Representative pictures of interphase, prophase, prometaphase, metaphase, and early and late anaphases are shown. Bar, $10 \mu \mathrm{m}$. For p1419 staining, see Supplemental Figure 6B.

Immunofluoresence microscopy using pT1415 and pS1419 demonstrated that these modifications take place specifically in mitosis, from prophase to early anaphase (Fig. 4E; Supplemental Fig. 6B). The finding that phosphorylation of Thr 1415 induces Plk1 to bind CAP-D3 raises the possibility that phosphorylation of Ser 1419 is facilitated by CAP-D3-bound Plk1. In line with this idea, we noticed that the chromosome staining with pS1419 antibodies is diminished in T1415A-replaced cells (Fig. 5A). Immunoblotting analysis also suggested that the levels of Ser 1419 phosphorylation were reduced without Thr 1415 phosphorylation (Fig. 5B, lane 14; Supplemental Fig. 6B, lane 8). These data support the notion that phosphorylation of Ser 1419 is efficiently catalyzed by CAP-D3-bound Plk1. In this experiment, we also noticed that the hyperphosphorylation of CAP-D3 is suppressed in T1415A-replaced cells (Fig. 5B, lane 4; Supplemental
Fig. 6B, lane 2), suggesting that phosphorylation of Thr 1415 is required for hyperphosphorylation of CAP-D3. Moreover, the finding that S1419A has little effect on hyperphosphorylation of CAP-D3 implies the existence of other Plk1-mediated phospho-sites (Fig. 5B, lane 5).

\section{Phosphorylation of CAP-D3 Thr 1415 triggers full phosphorylation of condensin II}

As the other condensin II subunits, CAP-G2 and CAP-H2, undergo Plk1-dependent phosphorylation (Fig. 1D), we asked whether phosphorylation of these subunits also relies on Thr 1415 phosphorylation (Fig. 6A). In mitotic extracts, CAP-G2 can be detected as two major bands, the upper being the hyperphosphorylated form (Figs. 1D, 6A [cf. lanes 1 and 2]). We found that the upper band was decreased in T1415A-replaced cells (Fig. 6A, lane 8), as in 
A
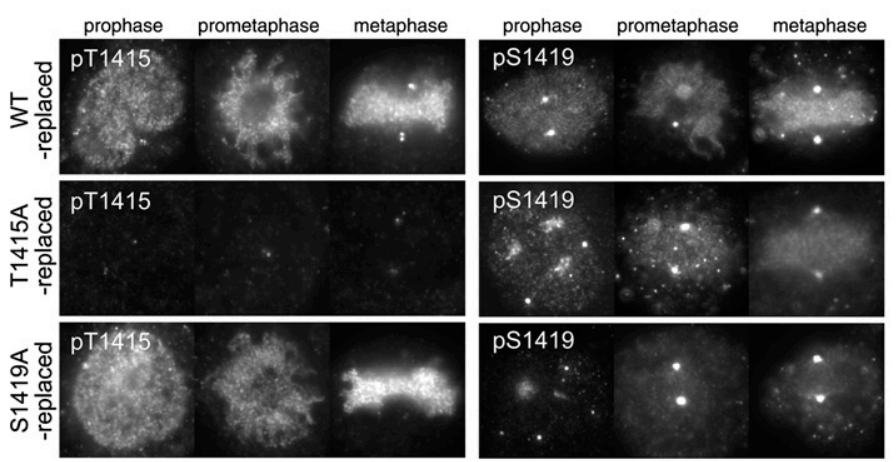

B
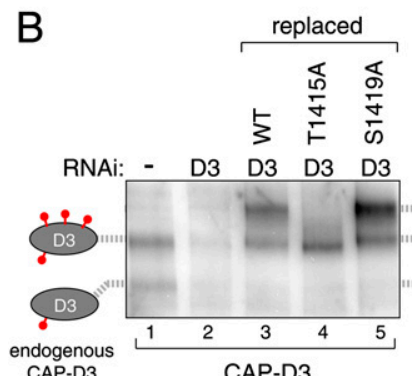

CAP-D3
CAP-D3
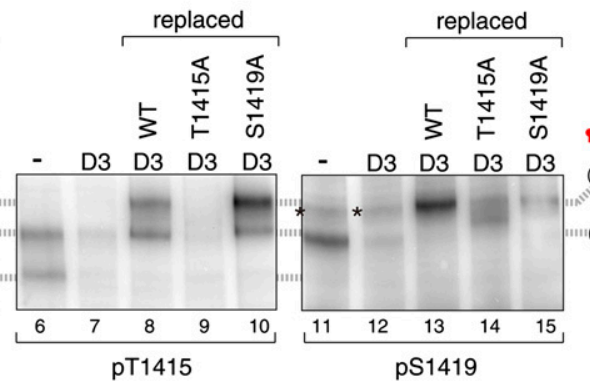

Figure 5. Hyperphosphorylation of CAP-D3 depends on CAP-D3-bound Plk1. (A) Reduced levels of Ser 1419 phosphorylation in T1415A-replaced cells. Cells in which endogenous CAP-D3 was replaced by either wildtype (top panels), T1415A (middle panels), or S1419A (bottom panels) forms of GFP-CAPD3 were fixed and stained with pT1415 or pS1419 antibodies. Note that pS1419 antibodies cross-react with an unidentified epitope at centrosomes, seen as two marked dots that do not disappear after depletion of CAPD3. Quantification of fluorescence intensities is summarized in Supplemental Figure 5, C and D. (B) Regulation of Thr 1415 and Ser 1419 phosphorylation. (First two lanes of each panel) Two species with different phosphorylation levels of endogenous CAP-D3 can be detected in mitotic cell extracts that diminish after CAP-D3 depletion, as depicted on the left side. (Last three lanes of each panel) Mitotic extracts from wild-type-replaced, T1415A-replaced or S1419A-replaced cells were analyzed. As replaced CAP-D3 proteins are tagged with GFP, they migrate slower than the endogenous proteins. The two corresponding phosphorylated forms of GFPCAP-D3 are positioned on the right side. Asterisks mark nonspecific bands, which do not appear in immunopurified GFP-CAP-D3 samples (Supplemental Fig. 6A). cells depleted of CAP-D3 or Plk1 (Fig. 6A, lanes 3,4). Likewise, the mitotic mobility retardation of CAP-H2 was mostly abolished in these three conditions (Fig. 6A, lanes $3,4,8)$. These results suggest that Plk1 bound to CAP-D3 facilitates hyperphosphorylation of all of the non-Smc subunits of condensin II. Of note, threonine residues in the C-terminal region of condensin I subunit CAP-D2 have been identified as a mitotic phosphorylation target of Cdk1 in frog extracts (Kimura et al. 1998). However, CAP-D2 does not seem to participate in recruiting Plk1, as depletion of CAP-D2 did not displace Plk1 from chromosomal axes (Fig. 3D).

Having been able to detect phosphorylations on CAPD3 in vivo, we wished to identify the kinases involved. A 20-min pretreatment of mitotic cells with a Cdk1 inhibitor abolished phosphorylation of Thr 1415, and pretreatment with a Plk1 inhibitor reversed Ser 1419 phosphorylation (Fig. 6B). Together with in vitro experiments (Fig. 2), these results suggest that phosphorylation of Thr 1415 and Ser 1419 depends primarily on Cdk1 and Plk1, respectively. The Ser 1419 phosphorylation was also reversed by inhibiting Cdk1 (Fig. 6B), consistent with the idea that phosphorylation of Thr 1415 is a crucial step to induce further Plk1-mediated phosphorylation of condensin II. Based on these observations, we propose that the Cdk1-mediated phosphorylation of CAP-D3 Thr 1415 creates a binding module for the PBD, and CAP-D3-bound Plk1 further promotes hyperphosphorylation of the whole condensin II complex, including CAP-D3 itself (Fig. 6C).

\section{Phosphorylation stimulates the activity of condensin II}

Finally, to investigate the consequences of condensin II phosphorylation, we carried out live-cell imaging analysis and monitored the behavior of chromosomes during mitotic progression (Fig. 7A,B). Similar to previous studies (Hirota et al. 2004; Ono et al. 2004), the initial phase of chromosome condensation typically became discernible $\sim 15$ min before NEBD and progressed during prophase. In CAP-D3-depleted cells, the condensation was delayed and appeared only a few minutes before NEBD. This defective chromosome condensation in prophase after CAP-D3 depletion was recovered by expressing wild-type CAP-D3. In contrast, the T1415A mutant failed to rescue the defect; cells largely lacked chromosome condensation during prophase, when it arose shortly before NEBD, as seen in CAP-D3-depleted cells.

To supplement these observations in live cells, we additionally assessed the extent of chromosome condensation in fixed-cell preparations. As shown previously (Hirota et al. 2004), inactivation of condensin II by CAP-D3 depletion caused a strong reduction in chromosome condensation in prophase. This reduction was sufficiently recovered by expressing either wild-type CAP-D3 or the S1419A mutant, but not the T1415A mutant (Supplemental Fig. 7). These experiments indicate that CAP-D3 phosphorylation at Thr 1415 is a key modification for condensin II to initiate chromosome condensation in prophase.

One chromosome morphology related to condensin II depletion is known as "curly" chromosomes (Ono et al. 
A

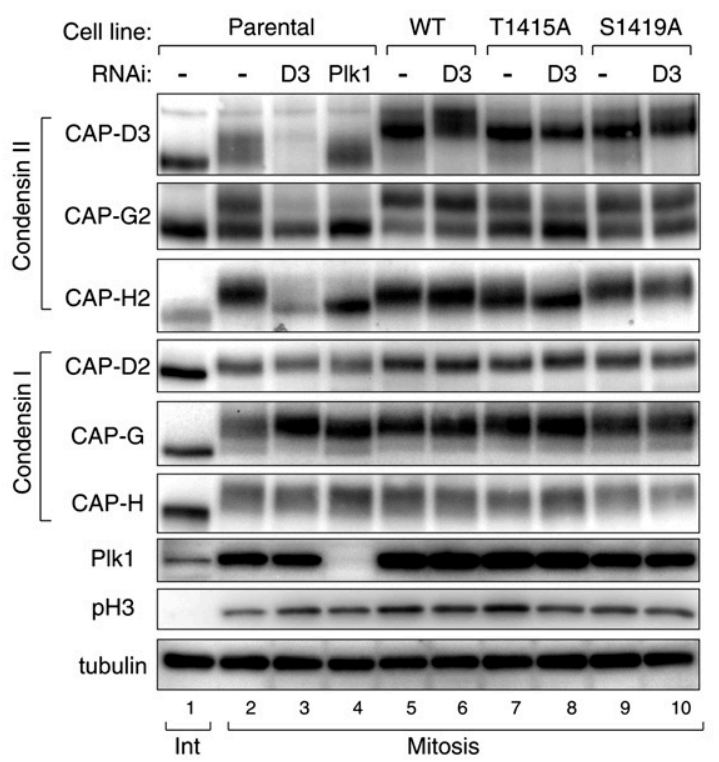

Figure 6. Cdk1-mediated phosphorylation of Thr 1415 is required for the full phosphorylation of condensin II. (A) Mitotic phosphorylation of non-Smc subunits of condensin II is perturbed in the T1415A mutant. Mitotic cell extracts prepared from indicated cell lines, with or without RNAi to Plk1 or to CAP-D3, were analyzed with the antibodies to condensin subunits. Parental cell line indicates the cell population that does not express any tagged protein. Note that changes in phosphorylation levels, as seen for condensin II subunits, are not readily detectable for condensin I subunits. (B) Phosphorylation of Thr 1415 and Ser 1419 depends primarily on Cdk1 and Plk1, respectively. Total cell extracts were prepared from thymidine-arrested interphase cells (lane 1) or nocodazolearrested mitotic cells (lanes 2-4) in which the activity of Cdk1 or Plk1 is inhibited by RO3306 or BI2536 treatment, respectively (lanes 3,4), and were analyzed by immunoblotting using the antibodies indicated. Note that pT1415 and pS1419 antibodies can hardly detect CAP-D3 protein in interphase cells (cf. lanes 1 and 2), indicating the specific reactivity of these antibodies to mitotic phosphorylated forms. (C) Illustrations depicting how condensin II complex is phosphorylated in mitosis, and how phosphorylations are affected in the T1415A and S1419A mutants. The model predicts the crucial role of Cdk1 in phosphorylating CAP-D3 at Thr 1415, which triggers the full phosphorylation of the condensin II complex, and explains why the phosphorylation levels are markedly decreased in T1415Areplaced but not S1419A-replaced cells.

2003). This characteristic structural change, emerging after treating cells with a hypotonic condition, is thought to reflect an improper chromosome assembly in the absence of condensin II. We found that $50 \%$ of the T1415Areplaced cells had chromosomes with the typical curly change, whereas in control wild-type-replaced cells, only $15 \%$ were affected. Importantly, $27 \%$ of the S1419Areplaced cells were found to have curly chromosomes, which implies that the Plk1-mediated phosphorylation of CAP-D3 is directly involved in mitotic chromosome assembly (Fig. 7D,E). Concomitantly, we found that T1415A-replaced cells were more susceptible to chromosome segregation failure than controls, as revealed by increased incidence of bridging or lagging chromosomes (Fig. 7C; Ono et al. 2004; Gerlich et al. 2006). The increased frequency of these errors was also seen in S1419A-replaced cells (Fig. 7C).

These results are consistent with the notion that phosphorylation of Thr 1415 and subsequent Plk1-mediated phosphorylation of CAP-D3 are essential for condensin II's function to properly assemble chromosomes and prevent segregation errors. Unlike T1415A-replaced cells, however, S1419A-replaced cells did not reveal overt defects in chromosome condensation in prophase (Fig. 7A,B; Supplemental Fig. 7). We suspect that this is because defects in chromosome condensation in prophase cells lacking a single Plk1 phosphorylation site were beyond the detection level of the light microscope. As Ser 1419 phosphorylation seems to already appear in prophase (Supplemental Fig. 6B), it is possible that chromosome assembly in prophase involves Plk1-mediated phosphorylation of condensin II.

\section{Discussion}

\section{CAP-D3 phosphorylation at Thr 1415 is crucial for condensin II function}

Mitotic chromosome assembly in prophase was largely suppressed in Thr 1415 nonphosphorylatable mutant cells, which indicates a key role of CAP-D3 phosphorylation on this particular site for the function of condensin II. In eukaryotic cells whose nuclear envelopes disassemble in mitosis, chromosome condensation seems to 
A
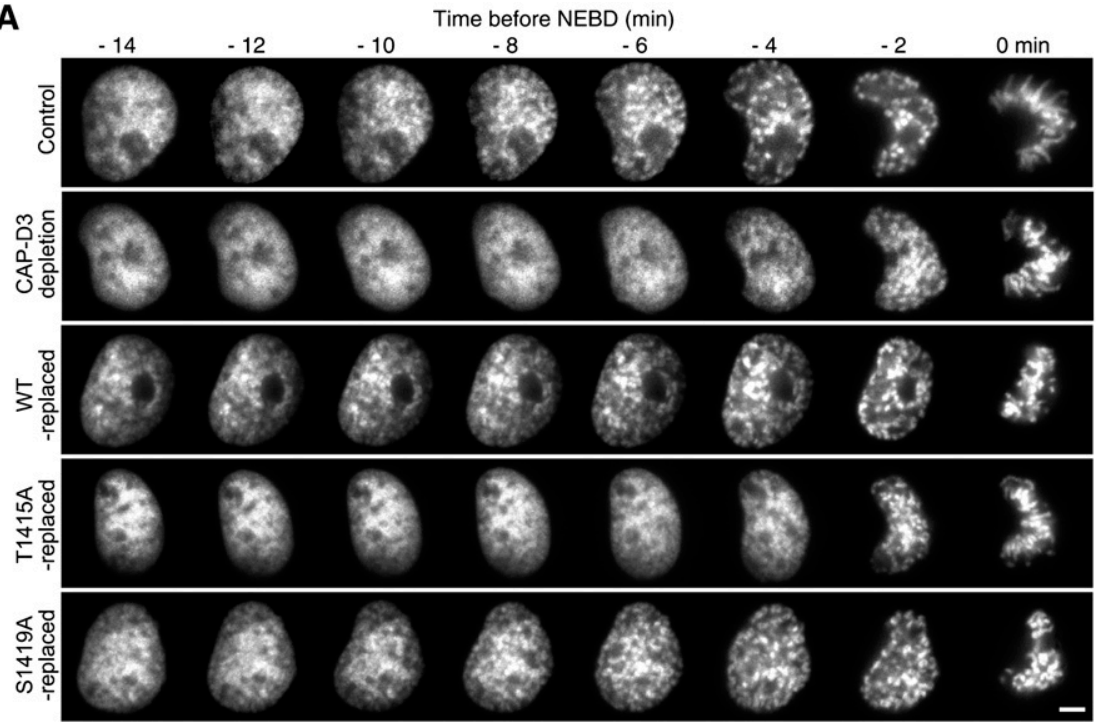

B

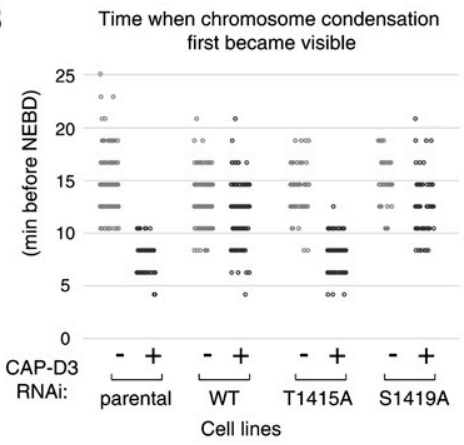

C

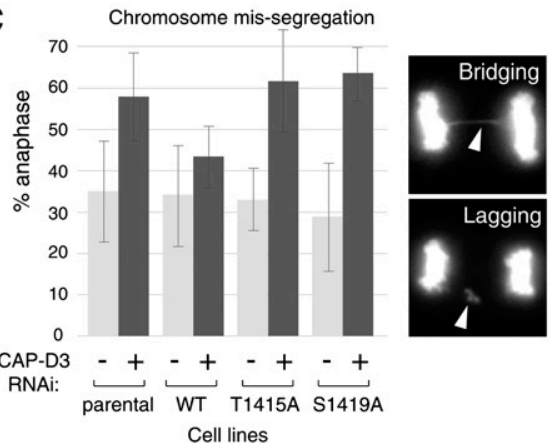

D
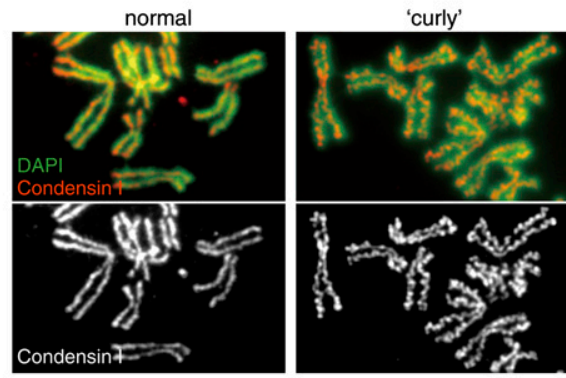

E

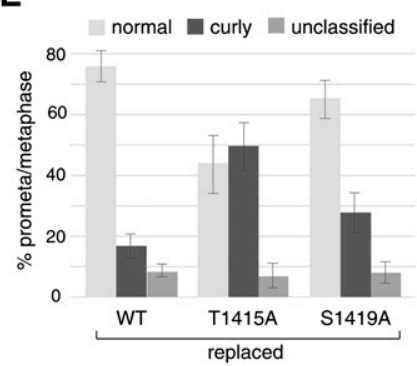

Figure 7. Nonphosphorylatable CAP-D3 mutants defective in mitotic functions of condensin II. (A) Analysis of the initial phases of chromosome condensation in live cells. Prophase image sequences were aligned on the time axis according to time before NEBD, which is defined by loss of a defined nuclear boundary. Bar, $5 \mu \mathrm{m}$. (B) Quantification of chromosome condensation in prophase. Time (minutes before NEBD) when chromosome condensation first became recognizable was scored and plotted for the indicated live-cell recordings. $(C)$ Analysis of defective chromosome segregation in anaphase in nonphosphorylatable CAP-D3 mutants. During live-cell imaging analysis, anaphase cells were assessed for the presence of lagging and/or bridging chromosomes, as exemplified in the panels on the right. Mean \pm SD from three experiment replicates ( $n=20 \sim 30$ cells per experiment) are shown in the histogram. Similar results were obtained in a fixed-cell analysis (Supplemental Fig. 8). (D) Examples of chromosome spreads demonstrating normal (left panels) or curly (right panels) appearance. Chromosome spreads were prepared from cells that had been treated with a hypotonic buffer and stained with CAP-H (a condensin I subunit) antibodies (red). DNA was counterstained with DAPI (green). (E) Abnormal curly change of chromosomal axes in nonphosphorylatable CAP-D3 mutants. More than 100 cells per indicated condition were examined and scored based on their chromosomal axis appearance (mean $\pm \mathrm{SD}$ ). initiate universally in prophase; i.e., before the NEBD. Our amino acid sequence conservation analysis identified a PBD-binding motif in conjunction with an adjacent prospective phospho-site in the C-terminal region of CAP-D3 protein in mammals as well as in worms and flies (Fig. 2C), but not in the budding/fission yeast CAPD3 counterparts Ycs4/Cnd1. An attractive hypothesis would be, therefore, that CAP-D3 phosphorylation at Thr 1415 or the equivalent site plays an essential role in activating condensin II, and thereby triggers chromosome condensation in prophase in all eukaryotes undergoing open mitosis.

In addition to the defective chromosome condensation in prophase, we found that chromosomes in the Thr 1415 mutant cells often reveal a curly appearance of chromo- somal axes and an increasing rate of chromosome segregation failure (Fig. 7). Taking into account a FRAP experiment that indicated that condensin II complexes bind stably to chromosomes throughout mitosis /Gerlich et al. 2006), condensin II complexes bearing the T1415A mutant possibly stay on chromosomes throughout mitosis once they are loaded during prophase. If so, we consider it plausible that the chromosomal phenotypes after NEBD are causally related to condensin II's inability to induce chromosome condensation in prophase.

\section{How does phosphorylation regulate condensin II?}

How might phosphorylation at Thr 1415 mechanistically stimulate the activity of condensin II? The mass 
spectrometric analysis pointed out multiple phosphosites on CAP-D3, and thus Thr 1415 would not be the only residue that is phosphorylated by Cdk1; still, our findings identify it as a key site that promotes further phosphorylation of the condensin II complex by Plk1. The Polo kinase-mediated phosphorylation of condensin has been shown to stimulate its supercoiling activity in budding yeast (St-Pierre et al. 2009). Thus, there is a precedent for the regulation of condensin via Plk1-mediated phosphorylation, and this mechanism may be conserved through evolution for the regulation of condensin II. Supporting this possibility, the S1419A mutation perturbed the ability of condensin II to induce mitotic chromosome assembly properly (Fig. 7C-E; Supplemental Fig. 8).

It is important to point out, however, that identification of Plk1 as a kinase for condensin II does not discount the possibility that phosphorylation by Cdk1 per se contributes to the activation of condensin II, as was originally proposed for condensin I (Kimura et al. 1998). The fact that chromosome condensation takes place before the NEBD in cells depleted of Plk1 activity (Lenart et al. 2007) allows us to hypothesize that phosphorylation of Cdk1 itself can stimulate the activity of condensin II, at least to some extent. Consistent with this view, in yeast, phosphorylation by Cdk 1 alone can partially stimulate the ability of condensin to induce supercoils (St-Pierre et al. 2009).

Analogous to condensin I, phosphorylation of condensin II might mediate the chromosomal localization of condensin II. However, we noticed that the nonphosphorylatable CAP-D3 mutants T1415A and S1419A were both found enriched at chromosome axes at levels comparable with the wild-type version (Fig. 4B), precluding the possibility that these phosphorylations by Cdk1 and Plk1 have a major role in the chromosomal association of condensin II. This interpretation is also consistent with the recent finding that chromosomal targeting of condensin II is mediated through the action of PP2A (Takemoto et al. 2009). Thus, instead of controlling the localization, we propose that the phosphorylation stimulates the activity of condensin II to promote mitotic chromosome assembly.

\section{Enrichment of Plk1 at chromosome axes}

A prominent feature of Plk1 is that this kinase changes its localization to various cellular structures during mitotic progression (including centrosomes, kinetochores, and the central spindle), which appears to be important to conduct specific functions at the right place (for review, see Petronczki et al. 2008; Archambault and Glover 2009). Our data are consistent with the model that Plk1 is recruited to chromosome axes along chromosome arms by binding to CAP-D3, and that CAP-D3-bound Plk1 further mediates phosphorylation of condensin II subunits. An obvious follow-up question is to ask whether the range of Plk1 activities is confined to the condensin II complex, or whether it can also promote phosphorylation of other chromosomal proteins. Surprisingly, when elec- trophoretic mobility retardation is used as a readout for protein phosphorylation, Plk1 does not seem to act on the closely related complex condensin I (Fig. 1D), which also localizes at chromosome axes, but this might simply be because condensin I is not a substrate for Plk1.

It would be interesting to find out next whether topoisomerase II $\alpha$ is controlled by the chromosomal axisenriched Plk1, as this enzyme becomes enriched at chromosome axes in prophase to promote chromosome assembly (Gimenez-Abian et al. 1995) and is found to undergo Plk1-dependent mitotic phosphorylation (Santamaria et al. 2011). However, we speculate that the function of topoisomerase II $\alpha$ to shorten chromatid lengths is not directly regulated by Plk1 on axes because we have never seen that condensin II depletion, a condition that displaces Plk1 from chromosome axis, resulted in abnormally long chromosomes (Hirota et al. 2004). These observations support the view that Plk1 has a very special mode of action in that it requires physical binding to its substrates to ensure efficient phosphorylation, and therefore the activity of the kinase can, in principle, reach to its bound protein or the protein complex to which Plk1 is bound. Such confined action of Plk1 contrasts with the properties of other mitotic kinases.

Plk1 has been implicated in the phosphorylation of the cohesin complex during the so-called "prophase pathway," the mechanism that removes cohesin complexes from chromosome arms in prophase (Losada et al. 2002; Sumara et al. 2002). An interesting question to ask is whether cohesin complexes enriched in between sister chromatids are targeted by CAP-D3-bound Plk1. However, the prophase pathway seems to be intact in cases in which Plk1 had been depleted or displaced from chromosome axes, because both dissociations of sister chromatid cohesion between chromosome arms seem to proceed normally under those conditions (Supplemental Fig. 9). How might Plk1 then act on cohesin on chromosome arms? Having found the confined action of Plk1 to condensin II, we consider it possible that Cdk1 phosphorylates one of the cohesin subunits and recruits Plk1, which enables an efficient phosphorylation of the cohesin complex. This hypothesis would provide an intriguing view of how Cdk1 activity promotes dissociation of sister chromatid cohesion on the chromosome arms, which proceeds in parallel to chromosome condensation in cells entering mitosis.

\section{Materials and methods}

\section{Antibodies}

Polyclonal rabbit antibodies to condensin complexes were raised by immunizing two peptides in combination for each subunit, as follows: CAP-D3 (CTKRAISTPEKSISD [corresponding amino acid sequence number 1409-1422] and CSRRSLRKTPLKTAN [1484-1498]), CAP-D2 (EFHLPLSPEELLKSC [7-20] and CTTPIL RASARRHR [1388-1400]), CAP-H2 (CRTNVDLKNDQTPSE [78-91] and CKRFQTYAAPSMAQP [543-556]), CAP-G2 (CGED NMETEHGSKMR [77-90] and CYESSSRTLGELLNS [11301143]), Smc4 (AGEKILGPFHKRFSC [10-24] and VAVNPKEIAS KGLC [1189-1202]). The resulting antisera were affinity-purified 
against the peptides used for immunization. Antibodies to Smc2 and CAP-H were generously provided by Jan-Michael Peters (Research Institute of Molecular Pathology, Vienna), and CAP-G is published in Nakajima et al. (2007). The phospho-specific antibodies pT1415 and pS1419 were raised against synthetic phosphopeptides CTKRAIS(pT)PEKSISDVTF and CTPEK(pS)ISDVT, respectively, in rabbits. The resulting antisera were subjected to sequential affinity purification on phospho-peptide and nonphospho-peptide columns. The following monoclonal mouse antibodies were used: Plk1 (F8, Santa Cruz Biotechnology), Cyclin B1 (clone 18, BD Biosciences), $\alpha$-tubulin (B512, Sigma), GFP (G1544, Sigma), Cdc27 (clone 35, BD Biosciences).

\section{Immunoblot and Far-Western analyses}

Cells were extracted in a buffer consisting of $20 \mathrm{mM}$ Tris- $\mathrm{HCl}$ $(\mathrm{pH} 7.4), 100 \mathrm{mM} \mathrm{NaCl}, 20 \mathrm{mM} \beta$-glycerophosphate, $5 \mathrm{mM}$ $\mathrm{MgCl}_{2}, 0.1 \%$ Triton X-100, $10 \%$ glycerol, and $1 \mathrm{mM}$ DTT, supplemented with a cocktail of protease inhibitors (Complete Mini EDTA-free, Roche Diagnostics). When looking for electrophoretic mobility shifts, extraction was performed in the presence of $100 \mathrm{nM}$ okadaic acid, $2 \mathrm{mM} \mathrm{Na}_{3} \mathrm{VO}_{4}$, and $10 \mathrm{mM} \mathrm{NaF}$. To control the protein amount loaded per lane, total protein concentration of cell lysates was measured by the Bradford method (Protein Assay System, Bio-Rad Laboratories). Immunoblotting was performed as described previously (Nakajima et al. 2007). The recombinant PBD protein was purified from bacteria expressing a GST-fused C-terminal fragment of Plk1 (spanning amino acid residues 305-903) that contains the PBD. Far-Western assays were performed with the recombinant GST-PBD protein $(20 \mu \mathrm{g} / \mathrm{mL})$ in Tris-buffered saline (TBS) containing $1 \%$ skim milk and $0.01 \%$ Triton X-100 for $3 \mathrm{~h}$ at $4{ }^{\circ} \mathrm{C}$, followed by detection of bound proteins with monoclonal Plk1 antibodies that recognize its C-terminal fragment.

\section{Immunoprecipitation}

Mitotic cells were lysed in immunoprecipitation buffer $(20 \mathrm{mM}$ Tris- $\mathrm{HCl}$ at $\mathrm{pH} 7.5,150 \mathrm{mM} \mathrm{NaCl}, 20 \mathrm{mM} \beta$-glycerophosphate, $5 \mathrm{mM} \mathrm{MgCl}_{2}, 0.1 \%$ NP-40, protease inhibitors [Complete Mini EDTA-free, Roche], $1 \mathrm{mM}$ DTT), supplemented with $100 \mathrm{nM}$ okadaic acid, $2 \mathrm{mM} \mathrm{Na}_{3} \mathrm{VO}_{4}, 10 \mathrm{mM} \mathrm{NaF}$, and $0.25 \mathrm{U} / \mathrm{L}$ benzonase nuclease (Novagen), for $20 \mathrm{~min}$ on ice. Cell extracts, after removing the insoluble fraction by centrifugation at 15,000 $\mathrm{rpm}$ for $30 \mathrm{~min}$ at $4^{\circ} \mathrm{C}$, were used for immunoprecipitation. Typically, $10 \mu \mathrm{L}$ of protein A (Bio-Rad) or protein G-Sepharose (GE Healthcare) beads coupled to antibodies were incubated with cell extracts for $2 \mathrm{~h}$ at $4^{\circ} \mathrm{C}$, then washed three times with immunoprecipitation buffer and three times with $0.05 \%$ TBSTween20. Mass spectrometry was performed as described (Nozawa et al. 2010). Dephosphorylation of immunoprecipitated condensin was performed on beads using $\lambda$-phosphatase (New England Biolabs) in a buffer containing $50 \mathrm{mM}$ Tris- $\mathrm{HCl}(\mathrm{pH}$ 7.5), $0.1 \mathrm{mM}$ $\mathrm{Na}_{2}$ EDTA, $5 \mathrm{mM} \mathrm{DTT}$, and $2 \mathrm{mM} \mathrm{MnCl}$ for $30 \mathrm{~min}$ at $30^{\circ} \mathrm{C}$.

\section{Cell synchronization, inhibitor treatment, and cell lines}

HeLa cells were cultured and synchronized with a doublethymidine block protocol as described previously (Nakajima et al. 2007). Mitotic cells were shaken off and collected from cell culture dishes that had been treated for $3 \mathrm{~h}$ with $50 \mathrm{ng} / \mathrm{mL}$ nocodazole, which was added at a $6 \mathrm{~h}$-time point after the release from the second thymidine block. These mitotic cells were then treated with $10 \mu \mathrm{M}$ RO3306 (Roche) or with an equivalent concentration of the solvent DMSO (mock) for 20 min before being harvested for immunoblot analyses. To inhibit Plk1 activity, $100 \mathrm{nM}$ BI2536 (Tocris) was added at the 6-h time point with nocodazole. To generate cell lines that stably express fluorescent-tagged proteins, plasmids encoding a series of AcGFP-CAP-D3 were transfected using FuGENE 6 reagent (Roche). Stably expressing cell clones were selected in a complete medium containing $0.2 \mu \mathrm{g} / \mathrm{mL}$ puromycin and verified by fluorescence microscopy and immunoblotting for the expression of the tagged transgene.

\section{$R N A i$}

The sequences of the siRNAs were as follows: CAP-D3, 5'-AGG AAUUCAAGUUAACAGAGGCUUG-3'; Plk1, 5' -CGCCCAAC CAUUAACGAGCUGCUUA-3'. The cells were transfected by incubating $50 \mathrm{nM}$ duplex siRNA with RNAi-MAX transfection reagent (Invitrogen) in antibiotic-free growth medium. RNAi treatment was performed concomitantly with the synchronization with thymidine; the length of substantial transfections with siRNA targeting CAP-D3 and Plk1 was $48 \mathrm{~h}$ and $24 \mathrm{~h}$, respectively. For control transfections, the same annealing reaction was set up using $\mathrm{H}_{2} \mathrm{O}$ instead of siRNA oligonucleotides.

\section{In vitro phosphorylation}

Recombinant GST-fused CAP-D3 fragments (amino acid sequence 1399-1429), including wild-type and mutants, were expressed in bacteria BL21 and purified using glutathione beads, and used as kinase substrates. Reactions were carried out for 30 min at $37^{\circ} \mathrm{C}$ in a kinase reaction buffer $(5 \mathrm{mM}$ MOPS at $\mathrm{pH} 7.2$, $2.5 \mathrm{mM} \beta$-glycerolphosphate, $5 \mathrm{mM} \mathrm{MgCl}_{2}, 1 \mathrm{mM}$ EGTA, $0.4 \mathrm{mM}$ EDTA, $100 \mathrm{ng} / \mu \mathrm{L}$ BSA, $10 \mathrm{mM}$ DTT, $1 \mathrm{mM} \mathrm{ATP,}$ $\left.1 \mu \mathrm{Ci} / \mu \mathrm{L}\left[\gamma_{-}{ }^{32} \mathrm{P}\right] \mathrm{ATP}\right)$. The protein kinases used were recombinant Cdk1/Cyclin B (Carna Bioscience) or Plk1 (Cell Signaling Technology). Phosphorylation of GST-fused CAP-D3 fragments was detected by autoradiography.

\section{Immunofluorescence microscopy}

Cells grown on coverslips were fixed with ice-chilled $100 \%$ methanol for $20 \mathrm{~min}$ at $-20^{\circ} \mathrm{C}$, or with $2 \%$ paraformaldehyde in $0.137 \mathrm{M}$ sodium phosphate buffer ( $\mathrm{pH}$ 7.4). In some cases, a preextraction protocol was carried out for $2 \mathrm{~min}$ in $0.1 \%$ Triton $\mathrm{X}-100-\mathrm{PBS}$, followed by a 3 -min incubation in PBS before fixation. Fixed cells were permeabilized with $0.2 \%$ Triton $\mathrm{X}-100-\mathrm{PBS}$ and incubated with $3 \% \mathrm{BSA} / \mathrm{PBS}$ for at least $1 \mathrm{~h}$. In some experiments, mitotic cells were shaken off, immersed in a hypotonic buffer (PBS:distilled water $=2: 3$ solution) for $5 \mathrm{~min}$, and spun onto glass slides at $1000 \mathrm{rpm}$ for $5 \mathrm{~min}$ using a cytospin centrifuge (Shandon). Cells were incubated with the primary antibodies overnight at room temperature, followed by incubation with secondary antibodies for $1 \mathrm{~h}$. The secondary antibodies used in this study were goat anti-rabbit IgG Alexa fluor 488 and 568, goat anti-mouse Alexa fluor 488 and 568, and goat antihuman IgG Alexa fluor 568 (Molecular Probes). After a 5-min incubation with $0.1 \mu \mathrm{g} / \mathrm{mL} \quad$ 4',6-diamidino-2-phenylindole (DAPI), cells were mounted in Prolong Gold anti-fade mounting reagent (Invitrogen). Images were acquired on a Zeiss Imager Z.1 microscope equipped with epifluorescence and a Photometrics Cool Snap HQ CCD camera.

\section{Live-cell imaging analysis}

Cells were placed into $\mathrm{CO}_{2}$-independent medium without phenol red (Gibco) on Lab-Tek chambered coverslips (Nunc), and the chamber lids were sealed with silicone grease. Images were captured every 2 min, with 100-msec exposure times, through a $100 \times / 1.40$ NA Plan Apochromat oil objective lens mounted on 
an inverted microscope (IX-71; Olympus) equipped with a Cool Snap HQ CCD camera (Photometrics). A series of projected images of five $Z$-sections with $4.0-\mu \mathrm{m}$ intervals were analyzed.

\section{Acknowledgments}

We are grateful to Keiji Kimura (Tsukuba University) for CAP-D3 cDNA, Jan-Michael Peters (IMP, Vienna) for CAP-H and Smc2 antibodies, Kazuki Kumada (JFCR Cancer Institute, Tokyo), and James Hutchins (IMP, Vienna) for crucial comments on the manuscript. Research in the laboratory of T.H. is supported by grants from the Japan Society for the Promotion of Science (JSPS) and the Ministry of Education, Culture, Sports and Technology of Japan (MEXT), and a research grant from the Cell Science Research Foundation.

\section{References}

Archambault V, Glover DM. 2009. Polo-like kinases: conservation and divergence in their functions and regulation. Nat Rev Mol Cell Biol 10: 265-275.

Elia AE, Rellos P, Haire LF, Chao JW, Ivins FJ, Hoepker K, Mohammad D, Cantley LC, Smerdon SJ, Yaffe MB. 2003. The molecular basis for phosphodependent substrate targeting and regulation of Plks by the Polo-box domain. Cell 115: 83-95.

Gerlich D, Hirota T, Koch B, Peters JM, Ellenberg J. 2006. Condensin I stabilizes chromosomes mechanically through a dynamic interaction in live cells. Curr Biol 16: 333-344.

Giet R, Glover DM. 2001. Drosophila aurora B kinase is required for histone $\mathrm{H} 3$ phosphorylation and condensin recruitment during chromosome condensation and to organize the central spindle during cytokinesis. J Cell Biol 152: 669-682.

Gimenez-Abian JF, Clarke DJ, Mullinger AM, Downes CS, Johnson RT. 1995. A postprophase topoisomerase II-dependent chromatid core separation step in the formation of metaphase chromosomes. J Cell Biol 131: 7-17.

Hagstrom KA, Holmes VF, Cozzarelli NR, Meyer BJ. 2002. C. elegans condensin promotes mitotic chromosome architecture, centromere organization, and sister chromatid segregation during mitosis and meiosis. Genes \& Dev 16: 729-742.

Hirano T. 2005. SMC proteins and chromosome mechanics: from bacteria to humans. Philos Trans $R$ Soc Lond B Biol Sci 360: $507-514$.

Hirano T. 2009. Let's play polo in the field of condensation. Mol Cell 34: 399-401.

Hirota T, Gerlich D, Koch B, Ellenberg J, Peters JM. 2004. Distinct functions of condensin I and II in mitotic chromosome assembly. J Cell Sci 117: 6435-6445.

Kaitna S, Pasierbek P, Jantsch M, Loidl J, Glotzer M. 2002. The aurora B kinase AIR-2 regulates kinetochores during mitosis and is required for separation of homologous chromosomes during meiosis. Curr Biol 12: 798-812.

Kimura K, Hirano M, Kobayashi R, Hirano T. 1998. Phosphorylation and activation of $13 \mathrm{~S}$ condensin by $\mathrm{Cdc} 2$ in vitro. Science 282: 487-490.

Kraft C, Herzog F, Gieffers C, Mechtler K, Hagting A, Pines J, Peters JM. 2003. Mitotic regulation of the human anaphase-promoting complex by phosphorylation. EMBO I 22: 6598-6609.

Lavoie BD, Hogan E, Koshland D. 2004. In vivo requirements for rDNA chromosome condensation reveal two cell-cycle-regulated pathways for mitotic chromosome folding. Genes \& Dev 18: 76-87.

Lenart P, Petronczki M, Steegmaier M, Di Fiore B, Lipp J, Hoffmann M, Rettig WJ, Kraut N, Peters JM. 2007. The small-molecule inhibitor BI 2536 reveals novel insights into mitotic roles of polo-like kinase 1. Curr Biol 17: 304-315.
Lipp JJ, Hirota T, Poser I, Peters JM. 2007. Aurora B controls the association of condensin I but not condensin II with mitotic chromosomes. J Cell Sci 120: 1245-1255.

Losada A, Hirano M, Hirano T. 2002. Cohesin release is required for sister chromatid resolution, but not for condensin-mediated compaction, at the onset of mitosis. Genes \& Dev 16: 3004-3016.

Nakajima M, Kumada K, Hatakeyama K, Noda T, Peters JM, Hirota T. 2007. The complete removal of cohesin from chromosome arms depends on separase. J Cell Sci 120: 4188-4196.

Nasmyth K, Haering CH. 2005. The structure and function of SMC and kleisin complexes. Annu Rev Biochem 74: 595-648.

Nigg EA. 1995. Cyclin-dependent protein kinases: key regulators of the eukaryotic cell cycle. Bioessays 17: 471-480.

Nozawa RS, Nagao K, Masuda HT, Iwasaki O, Hirota T, Nozaki N, Kimura H, Obuse C. 2010. Human POGZ modulates dissociation of HP1 $\alpha$ from mitotic chromosome arms through Aurora B activation. Nat Cell Biol 12: 719-727.

Nurse P. 1990. Universal control mechanism regulating onset of M-phase. Nature 344: 503-508.

Ono T, Losada A, Hirano M, Myers MP, Neuwald AF, Hirano T. 2003. Differential contributions of condensin I and condensin II to mitotic chromosome architecture in vertebrate cells. Cell 115: 109-121.

Ono T, Fang Y, Spector DL, Hirano T. 2004. Spatial and temporal regulation of Condensins I and II in mitotic chromosome assembly in human cells. Mol Biol Cell 15: 3296-3308.

Petersen J, Hagan IM. 2003. S. pombe aurora kinase/survivin is required for chromosome condensation and the spindle checkpoint attachment response. Curr Biol 13: 590-597.

Petronczki M, Lenart P, Peters JM. 2008. Polo on the rise-from mitotic entry to cytokinesis with Plk1. Dev Cell 14: 646-659.

Santamaria A, Neef R, Eberspacher U, Eis K, Husemann M, Mumberg D, Prechtl S, Schulze V, Siemeister G, Wortmann $\mathrm{L}$, et al. 2007. Use of the novel Plk1 inhibitor ZK-thiazolidinone to elucidate functions of Plk1 in early and late stages of mitosis. Mol Biol Cell 18: 4024-4036.

Santamaria A, Wang B, Elowe S, Malik R, Zhang F, Bauer M, Schmidt A, Silljé HHW, Körner R, Nigg EA. 2011. The Plk1dependent phosphoproteome of the early mitotic spindle. Mol Cell Proteomics 10: M110.004457. doi: 10.1074/mcp. M110.004457.

St-Pierre J, Douziech M, Bazile F, Pascariu M, Bonneil E, Sauve V, Ratsima H, D'Amours D. 2009. Polo kinase regulates mitotic chromosome condensation by hyperactivation of condensin DNA supercoiling activity. Mol Cell 34: 416-426.

Sumara I, Vorlaufer E, Stukenberg PT, Kelm O, Redemann N, Nigg EA, Peters JM. 2002. The dissociation of cohesin from chromosomes in prophase is regulated by Polo-like kinase. Mol Cell 9: 515-525.

Takemoto A, Murayama A, Katano M, Urano T, Furukawa K, Yokoyama S, Yanagisawa J, Hanaoka F, Kimura K. 2007. Analysis of the role of Aurora B on the chromosomal targeting of condensin I. Nucleic Acids Res 35: 2403-2412.

Takemoto A, Maeshima K, Ikehara T, Yamaguchi K, Murayama A, Imamura S, Imamoto N, Yokoyama S, Hirano T, Watanabe $\mathrm{Y}$, et al. 2009. The chromosomal association of condensin II is regulated by a noncatalytic function of PP2A. Nat Struct Mol Biol 16: 1302-1308.

Yeong FM, Hombauer $\mathrm{H}$, Wendt KS, Hirota $\mathrm{T}$, Mudrak I, Mechtler K, Loregger T, Marchler-Bauer A, Tanaka K, Peters JM, et al. 2003. Identification of a subunit of a novel Kleisin$\beta /$ SMC complex as a potential substrate of protein phosphatase 2A. Curr Biol 13: 2058-2064. 


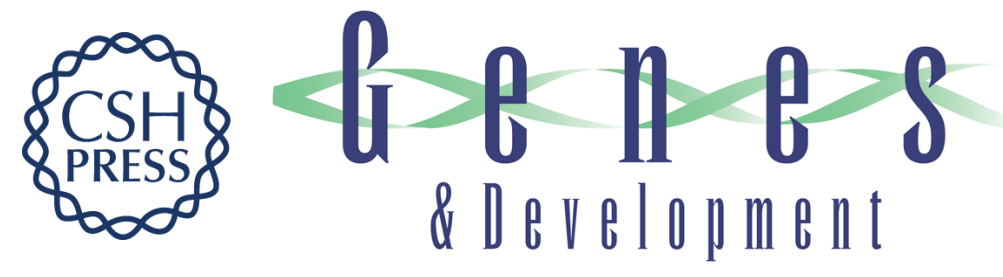

\section{The initial phase of chromosome condensation requires Cdk1-mediated phosphorylation of the CAP-D3 subunit of condensin II}

Satoshi Abe, Kota Nagasaka, Youko Hirayama, et al.

Genes Dev. 2011, 25:

Access the most recent version at doi:10.1101/gad.2016411

Supplemental http://genesdev.cshlp.org/content/suppl/2011/04/12/25.8.863.DC1
Material

References This article cites 32 articles, 13 of which can be accessed free at: http://genesdev.cshlp.org/content/25/8/863.full.html\#ref-list-1

License

Email Alerting Receive free email alerts when new articles cite this article - sign up in the box at the top Service right corner of the article or click here.

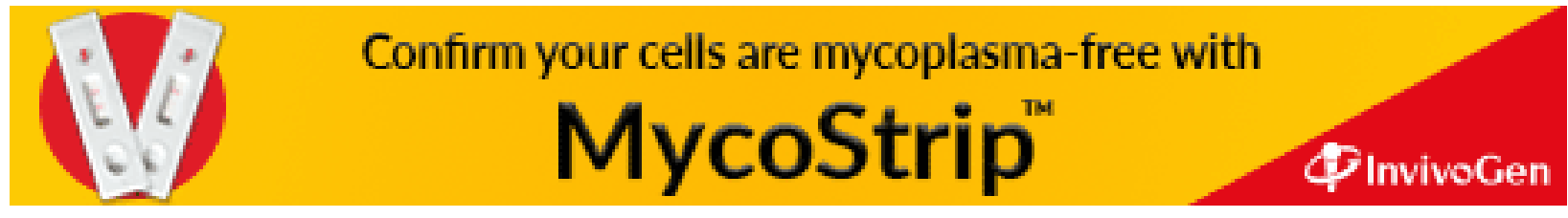

